\title{
CAUSAL ACTION-AT-A-DISTANCE INTERPRETATION OF THE ASPECT-RAPISARDA EXPERIMENTS
}

\author{
Nicola CUFARO PETRONI \\ Istituto di Fisica dell'Università, Bari, Italy \\ and INFN Sezione di Bari, Bari, Italy \\ and \\ Jean-Pierre VIGIER \\ Equipe de recherche associée au CNRS no 533, Institut H. Poincaré, 75231 Paris, France
}

Received 15 September 1982

Recent results in EPR-type experiments on singlet photon pairs which establish (a) the non-existence of local hidden variables, (b) the existence of quantum superluminal correlations between the action of independent parts of a measuring device separated by space-like intervals, are interpreted causally within the frame of the stochastic interpretation of quantum mechanics.

Physicists who support Einstein's view in the Bohr -Einstein controversy are now confronted with the challenge of interpreting in a causal way the non-local result just established by Aspect et al. [1] (see also refs. $[2,3]$ ) and the eventual confirmation of that test in the forthcoming Rapisarda [4] experiment. To do this we start with a non-zero mass photon model. This is justified: (1) by the well-known fact [5-8] that the zero-mass limit of a non-zero mass spin-1 Proca particle cannot be physically distinguished from a Maxwell wave since the so-called transverse waves just correspond to $J_{3}= \pm 1$ (i.e. to opposite circular polarizations) while the longitudinal solutions $J_{3}=0$ (practically decoupled from transverse waves when $m_{\gamma} \rightarrow 0$ ) describe the Coulomb field when $m_{\gamma} \rightarrow 0$; (2) by the theoretical result that (with $m_{\gamma} \neq 0$ ) one has found a classical counterpart (i.e. the Weyssenhoff particle) to the photon field [9] , so that one can determine a classical counterpart of spin for isolated "classical" photons which is distributed [10] in the hydrodynamical representation of the Proca wave equation.

Both in the usual quantum-mechanical theory [11] and in the stochastic interpretation of quantum mechanics $[12,13]$ a system of two correlated photons $\left(m_{\gamma} \neq 0\right)$ can be represented by a second-rank tensor $A_{\mu \nu}$. As one knows this compound state of two spin-1 particles can be split [as a consequence of the grouprepresentation relation $D(1) \otimes D(1)=D(2) \oplus D(1)$ $\oplus D(0)$ ] into a symmetric part $A_{\mu \nu}$, a skew part $A_{\mu \nu}$ and a trace $A_{\mu \mu}$ representing respectively the $J=2, J$ $=1$, and $J=0$ compound states. Since the aforementioned experiments utilize $0-1-0$ singlet-state cascades we shall limit ourselves to the $A_{\mu \mu}, D(0), J=0$ singlet case.

Denoting by 1 and 2 the two photons (with coordinates $x_{1}^{\mu}$ and $x_{2}^{\mu}$ ) we represent our compound state by a scalar field

$$
\begin{aligned}
& \Phi\left(x_{1}, x_{2}\right)=A_{1 \mu}\left(x_{1}\right) A_{2}^{\mu}\left(x_{2}\right) \\
& \quad=\exp \left[R\left(x_{1}, x_{2}\right)+\mathrm{i} S\left(x_{1}, x_{2}\right)\right],
\end{aligned}
$$

where $\hbar=c=1$. As one knows [14] such a scalar field satisfies the system of relations

$\left(\square_{1}+\square_{2}-2 m_{\gamma}^{2}\right) \Phi=0,\left(\square_{1}-\square_{2}\right) \Phi=0$,

or equivalently

$\left(\square_{1}-m_{\gamma}^{2}\right) \Phi=0, \quad\left(\square_{2}-m_{\gamma}^{2}\right) \Phi=0$, 
along with the transverse gauge conditions $\partial_{1 \mu} A_{1}^{\mu}$ $=\partial_{2 \mu} A_{2}^{\mu}=0$, the second relation of (1) representing the causality constraint in the so-called predictive mechanics with action-at-a-distance [14]. This system is clearly deterministic in the sense that we shall now show that:

- the system of two $J=1$ particles can be solved in the forward (or backward) time direction in the sense of the Cauchy problem;

- the paths of the two particles are time-like;

- the formalism is invariant under the Poincare group $P=T \otimes \mathcal{L} \uparrow$.

Indeed writing $P_{i}^{\mu}=\partial S / \partial q_{i \mu}(i=1,2)$ we can split internal from external variables by writing $P^{\mu}=p_{1}^{\mu}+p_{2}^{\mu}$; $y^{\mu}=\frac{1}{2}\left(p_{1}^{\mu}-p_{2}^{\mu}\right) ; Q^{\mu}=\frac{1}{2}\left(q_{1}^{\mu}+q_{2}^{\mu}\right) ; z^{\mu}=q_{1}^{\mu}-q_{2}^{\mu}$; $q_{i}^{\mu}$ and $p_{i}^{\mu}$ representing pairs of canonical variables. Splitting (2) in to real and imaginary parts we obtain for the real parts

$\frac{1}{2}\left(\partial_{1 \mu} S \partial_{1}^{\mu} S\right)+U_{1}=\frac{1}{2} m_{\gamma}^{2}$

$\frac{1}{2}\left(\partial_{2 \mu} S \partial_{2}^{\mu} S\right)+U_{2}=\frac{1}{2} m_{\gamma}^{2}$

where we have $U_{i}=-\frac{1}{2}\left(\square_{i} R+\partial_{i}^{\mu} R \partial_{i \mu} R\right)$. This separation can be performed in the rest frame of the center of mass of the two photons where we consider the case of an eigenstate of $P_{\mu}$, i.e.

$\Phi=\varphi\left(z^{\mu}\right) \exp \left[\mathrm{i} k_{\mu}\left(x_{1}^{\mu}+x_{2}^{\mu}\right) / 2\right]$,

where $k^{\mu}$ is a constant time-like vector. In that case we have $\left(\partial_{1}^{\mu}+\partial_{2}^{\mu}\right) R=0$ and $\left(\partial_{1}^{\mu}+\partial_{2}^{\mu}\right) S=k^{\mu}$ so that subtracting eq. (3) we get $k^{\mu} \partial R / \partial z^{\mu}=0$ and hence $R$ only depends on $z_{\perp}^{\mu}=z^{\mu}-z_{\nu} k^{\nu} k^{\mu} / k^{2}$. In order to satisfy the condition $\{y \cdot P, U\}=0$ for the existence of causal time-like world lines [14] we must now make the substitution $z_{\perp}^{\mu} \rightarrow \tilde{z}^{\mu}=z^{\mu}+z_{\nu} P^{\nu} P^{\mu} / P^{2}$ so that $\left(\partial_{1}^{\mu}+\partial_{2}^{\mu}\right) R=0$ and $U_{1}=U_{2}=U\left(\tilde{z}_{\mu}\right)$. In that case the relations (3) represent a pair of causally bound photons connected by a causal action-at-a-distance. Moreover:

(i) The causality condition $P \cdot y=0$ implies that the Poisson bracket of the two photon hamiltonians $\left\{H_{1}\right.$, $\left.\mathrm{H}_{2}\right\}$ is zero, i.e. their corresponding proper times $\tau_{1}$ and $\tau_{2}$ are independent.

(ii) $q_{i}^{\mu}=x_{i}^{\mu}$ in the rest frame of the center of mass $\Sigma_{0}\left(k_{j}=0\right)$.

(iii) Subtracting eq. (3) with $U_{1}=U_{2}$ we get [15] $P \cdot y=0$ so that $\dot{P}_{\mu}=0$ where the dot denotes the operation $\frac{1}{2}\left(\partial / \partial \tau_{1}+\partial / \partial \tau_{2}\right)$. This yields $P \cdot \dot{y}=0$ which shows that no energy can be exchanged between the photons in $\Sigma_{0}$, so that no causal anomaly results from this particular type of action-at-a-distance.

(iv) We have $\dot{p}_{i} \cdot P=0$ so that the paths of both photons remain time-like.

(v) The formalism shows [16] that our causal covar. iant action-at-a-distance is instantaneous only in $\Sigma_{0}$ and its velocity $\eta$ can thus be calculated in any other frame $\Sigma$ by the corresponding $\Sigma_{0} \rightarrow \Sigma$ Lorentz transformation. In the particular case of the AspectRaspisarda experiment this immediately yields [16] $\eta=7.57 \mathrm{c}$ in the laboratory frame.

We now come to our interpretation of the Aspect -Rapisarda experiments.

Despite the confirmation [17] of quantum predictions in EPR experiments (i.e. the violation of Bell's inequalities [18]) a supplementary device was needed to prove directly the non-local character of this quantum correlation [19]. This set-up essentially rests on the use of calcite crystals acting as random switches on the photon paths which orient them, with probability $\frac{1}{2}$, in the $O$ (ordinary) or E (extraordinary) rays. The photons thus pick at random four possible paths and are subsequently detected through two pairs of linear polarizers $\mathrm{L}, \mathrm{L}^{\prime}, \mathrm{N}, \mathrm{N}^{\prime}$ with polarization directions $A$ and $B$ perpendicular to the $x$ axis (see fig. 1). In the last experiment [2] $\mathrm{L}\left(\mathrm{L}^{\prime}\right)$ and $\mathrm{N}\left(\mathrm{N}^{\prime}\right)$ are separated $12 \mathrm{~m}$. In the $m_{\gamma} \neq 0$ formalism the vector potentials are the field states $A_{i}^{\mu}$, and represent the photon polarization states. Since we assume them to be transverse $\left(A_{1 \mu} \partial_{1}^{\mu} S=A_{2 \mu} \partial_{2}^{\mu} S=0\right)$ we know [20] that a photon impinging normally upon a linear polarizer either passes or is stopped, thus answering yes or no ( 1 or 0$)$ to the question: "is your linear polarization found parallel or perpendicular to the direction $A(B)$ of the polarizer?".

Considering now a pair of singlet photons issuing from a cascade source $S$ we compute in a system where the photon pairs move in opposite directions

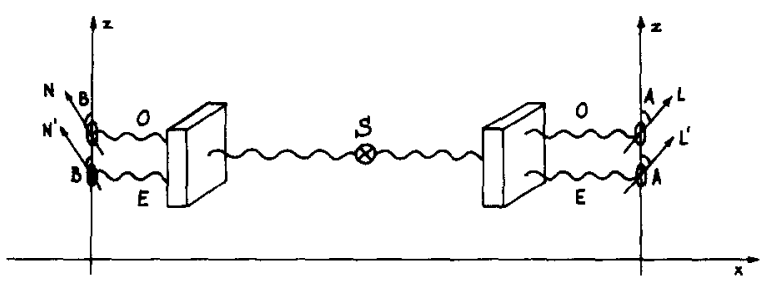

Fig. 1. Schematic view of the Aspect-Rapisarda experiment. 
parallel to the same axis $x$ and are detected in two linear polarizers. If we then set $\alpha=A-B$ we can compute the probability of the four possible answers to the composite question: "does photon 1 pass the polarizer $\mathrm{L}\left(\mathrm{L}^{\prime}\right)$ and photon 2 the polarizer $\mathrm{N}\left(\mathrm{N}^{\prime}\right)$ ?". The initial state is now represented by $A_{1 \mu} A_{2}^{\mu}$; since the $A_{i}^{\mu}$ are orthogonal to the $x$ axis we get

$|i\rangle=2^{-1 / 2}\left(\left|y_{1}\right\rangle\left|y_{2}\right\rangle+\left|z_{1}\right\rangle\left|z_{2}\right\rangle\right)$

in terms of state vectors polarized along two orthogonal axes in the $x=$ const. plane. Denoting by $(1,1)$, $(0,0),(1,0),(0,1)$ the probabilities of the four possible answers we can easily calculate them: for example $(1,1)$ is given if we consider that the final state is

$$
\begin{aligned}
|\mathrm{f}\rangle & =\left(\cos A\left|y_{1}\right\rangle+\sin A\left|z_{1}\right\rangle\right) \\
& \times\left(\cos B\left|y_{2}\right\rangle+\sin B\left|z_{2}\right\rangle\right),
\end{aligned}
$$

so that we have

$$
\begin{aligned}
(1,1) & =|\langle\mathrm{i} \mid \mathrm{f}\rangle|^{2}=\frac{1}{2}(\cos A \cos B+\sin A \sin B)^{2} \\
& =\frac{1}{2} \cos ^{2} \alpha
\end{aligned}
$$

and we get in an analogous way

$(0,0)=\frac{1}{2} \cos ^{2} \alpha, \quad(1,0)=(0,1)=\frac{1}{2} \sin ^{2} \alpha$.

The recent experimental confirmation [2] of this well-known quantum prediction implies, following Bell's work [18] and a recent analysis of Feynman [21], that there is no way in which a local hiddenvariable theory can reproduce such quantum correlations. Indeed, as another example [21], the classical local prediction for this probability for $\alpha=30^{\circ}$ in a typical arrangement is $2 / 3$ and is strikingly different from the quantum prediction of $3 / 4$ with which experiment agrees.

This situation can be interpreted in two ways. The first is that in the Copenhagen interpretation the two photons do not possess hidden polarizations when they leave the cascade and borrow their polarizations when they interact with the polarizers: the EPR paradox being that they are then non-locally correlated. To paraphrase Einstein's famous statement: "If God plays dices in that situation, then there is a space-like correlation which connects the two dices not when they are shaken in the cup, but at $\mathrm{L}\left(\mathrm{L}^{\prime}\right)$ and $\mathrm{N}\left(\mathrm{N}^{\prime}\right)$ when they stop rolling on the table". The second is that this result does not imply that the photons of each pair do not possess hidden polarizations but only that they are correlated by the quantum-potential action at a distance. This later statement will be clarified by the subsequent hydrodynamical analysis.

In the stochastic interpretation of quantum mechanics we start from the physical existence of de Broglie's waves [22] and we will show that in the hydrodynamical-stochastic representation of our experiments the spins (and polarizations) of the two photons are always defined and non-locally connected. Indeed if the total state $\Phi\left(x_{1}, x_{2}\right)$ satisfies eqs. (1) and (2) $[12,13]$ the lagrangian of our pair will be

$\mathcal{L}=m_{\gamma}^{2} \Phi^{*} \Phi+\partial_{1 \mu} \Phi^{*} \partial_{1}^{\mu} \Phi+\partial_{2 \mu} \Phi^{*} \partial_{2}^{\mu} \Phi$.

A classical relativistic hydrodynamical analysis [10, 23] then allows one to build the energy-momentum tensor for each single photon (from now on, because of the $1 \leftrightarrow 2$ symmetry, we will calculate only the quantities relative to photon (1), i.e.:

$$
\begin{gathered}
t_{1 \mu \nu}=\left[\partial \mathcal{L} / \partial\left(\partial_{1}^{\nu} A_{1}^{\lambda}\right)\right] \partial_{1 \mu} A_{1}^{\lambda}+\text { c.c. }-\mathscr{L} \delta_{\mu \nu} \\
=\partial_{1 \mu} \Phi^{*} \partial_{1 \nu} \Phi+\partial_{1 \mu} \Phi \partial_{1 \nu} \Phi^{*}-\mathscr{L} \delta_{\mu \nu} .
\end{gathered}
$$

From Belinfante's tensor [23]

$$
\begin{aligned}
& f_{1 \mu \nu \lambda}=\left[\partial \mathcal{L} / \partial\left(\partial_{1}^{\lambda} A_{1}^{\rho}\right)\right] \mathscr{I}_{\mu \nu}^{\rho \sigma} A_{1 \sigma}+\text { c.c. } \\
& \quad=\frac{1}{2}\left(\partial_{1 \lambda} \Phi^{*}\right)\left(A_{2 \mu} A_{1 \nu}-A_{1 \mu} A_{2 \nu}\right)+\text { c.c. },
\end{aligned}
$$

where $\mathscr{I}_{\mu \nu}^{\rho \sigma}=\frac{1}{2}\left(\delta_{\rho \mu} \delta_{\sigma \nu}-\delta_{\rho \nu} \delta_{\sigma \mu}\right)$, the spin density tensor becomes (if $u_{i}^{\mu}$ are the unitary four velocities of the photons)

$$
\begin{aligned}
& \frac{1}{2} S_{1 \mu \nu}=-u_{1}^{\lambda} f_{1 \mu \nu \lambda} \\
& \quad=\left(A_{1 \mu} A_{2 \nu}-A_{1 \nu} A_{2 \mu}\right) u_{1}^{\lambda} \partial_{1 \lambda} \Phi^{*}+\text { c.c. }
\end{aligned}
$$

and the spin vector can be written

$S_{1 \mu}=\frac{1}{2} \mathrm{i} \epsilon_{\nu \alpha \beta \mu} u_{1}^{\nu} S_{1}^{\alpha \beta}$.

Moreover, denoting now by a dot the derivative along a current line, we can show that because of the $t_{1 \mu \nu}$ symmetry we have [23]

$\dot{S}_{1 \mu \nu}=\partial_{1 \lambda}\left(u_{1}^{\lambda} S_{1 \mu \nu}\right)=t_{1 \mu \nu}-t_{1 \nu \mu}=0$.

From this it results that:

(1) $S_{1 \mu}$ has a constant length in the sense that $\dot{S}_{1}^{2}$ $=0$; indeed we see that [23] $S_{1}^{2}=S_{1 \mu} S_{1}^{\mu}=\frac{1}{2} S_{1 \alpha \beta} S_{1}^{\alpha \beta}$ because of the properties of $\epsilon_{\alpha \mu \nu \beta}$, the antisymmetry of $S_{1 \mu \nu}, u_{1}^{\mu} u_{1 \mu}=-1$, and $u_{1 \mu} A_{1}^{\mu}=u_{1 \mu} A_{2}^{\mu}=0$. Hence 
$\dot{S}_{1}^{2}=0$ because we showed that $S_{1 \mu \nu}=0$.

(2) The derivative of $S_{1}$ is $\dot{S}_{1}=\frac{1}{2} \mathrm{i} \epsilon_{\nu \alpha \beta \mu} S_{1}^{\alpha \beta}$ $\times\left(u_{1}^{\lambda} \partial_{1 \lambda} u_{1}^{\nu}\right)$ so that it depends in a non-local way on the $A_{2}\left(x_{2}\right)$ contained in $S_{1}^{\mu \nu}$.

(3) The elements of the photon pairs interact permanently not by exchanging tachyons but through action-at-a-distance which reflects the disturbance of Dirac's covariant stochastic aether [24] . In present experiments the photons are "holding hands" over $12 \mathrm{~m}$ and any disturbance of one of them is carried superluminally to the other by a phase-like disturbance of the stochastic quantum potential ... which includes a quantum torque. This interpretation of the EPRBohm experiments is identical to the one recently developed by Philippidis and Bohm to interpret the Aharonov-Bohm effect [25].

To conclude we are now confronted with the reality of non-local correlations between independent parts of a measuring device since the action of the polarizers $\mathrm{L}\left(\mathrm{L}^{\prime}\right)$ and $\mathrm{N}\left(\mathrm{N}^{\prime}\right)$ are space-like separated, and can be associated to four independent sets of observers and observations. In that case one can take three different attitudes:

(a) Assume that this brings nothing new to the present interpretation of quantum theory ... which is thus non-local by nature, as now confirmed by experiments. This no-problem attitude ignores the facts that (1) the instantaneous collapse of the wave packet by experiments in all frames is not a relativistic concept, (2) the instantaneous correlation between the observations of independent observers evidently goes beyond the present interpretations of the quantum measurement theory and (3) there is now a basic experimental irreducible discrepancy between any form of local classical causal interpretation and the results of quantum statistics in EPR experiments [18,21] .

(b) Assume that the Aspect-Rapisarda experiments raise new problems of interpretations which imply an extension of the present theory in the Copenhagen direction as suggested by Wigner [26] , Stapp [27] and Costa de Beauregard [20].

(c) Assume (as done by the authors and by Bohm and Hiley [28]) that one must interpret the new result in terms of a non-local quantum potential. This, of course, implies a real physical existence of de Broglie's waves. It is interesting to note that this line of thought has recently led to concrete experimental proposals which imply conflicting predictions
[29] between the Copenhagen and the causal stochastic interpretation of quantum mechanics ... identical in this case to Maxwell's predictions. In other words, we feel that one will only be able to assess the real meaning and theoretical implications of the AspectRapisarda experiments when the question of the real existence of de Broglie's waves will be settled (as is now attempted by Gozzini [30] in Pisa) by experimental physicists.

The authors wish to thank Professors Gozzini and Selleri and Dr. Droz-Vincent for helpful discussions in the preparation of this work. One of us (N.C.P.) is grateful to the Italian M.P.I. for a grant which made this research possible.

\section{References}

[1] A. Aspect, P. Grangier and G. Roger, Phys. Rev. Lett. 49 (1982) 91 .

[2] A. Aspect, P. Grangier and G. Roger, Phys. Rev. Lett. 47 (1981) 460

[3] D. Bohm, Phys. Rev. 85 (1952) 166, 180; 89 (1953) 458; D. Bohm and J.P. Vigier, Phys. Rev. 96 (1954) 208; 109 (1958) 1882;

E. Nelson, Phys. Rev. 150 (1966) 1079;

W. Lehr and J. Park, J. Math. Phys. 18 (1977) 1235;

F. Guerra and P. Ruggiero, Lett. Nuovo Cimento 23 (1978) 529;

J.P. Vigier, Lett. Nuovo Cimento 24 (1979) 258, 265; N. Cufaro Petroni and J.P. Vigier, Phys. Lett. $73 \mathrm{~A}$ (1979) 289; 81A (1981) 12;

Kh. Namsrai, Found. Phys, 10 (1980) 353, 731

[4] F. Falciglia, G. Iaci and V.A. Rapisarda, Lett. Nuovo Cimento 26 (1979) 327;

L. Pappalardo and V.A. Rapisarda, Lett. Nuovo Cimento 29 (1980) 221;

A. Garuccio and V.A. Rapisarda, Nuovo Cimento A65 (1981) 269.

[5] L. de Broglie, La méchanique ondulatoire du photon (Paris, 1940).

[6] L. Bass and E. Schrödinger, Proc. R. Soc. London A232 (1955) 1.

[7] S. Deser, Ann. Inst. Henri Poincaré 16 (1972) 79.

[8] M. Moles and J.P. Vigier, C.R. Acad. Sci. B276 (1973) 697.

[9] F. Halbwachs, F. Piperno and J.P. Vigier, Lett. Nuovo Cimento 33 (1982) 311.

[10] A. Garuccio and J.P. Vigier, Lett. Nuovo Cimento 30 (1981) 57.

[11] L.D. Landau and E.M. Lifshitz, Teoria quantistica relativistica (Rome, 1978).

[12] N. Cufaro Petroni and J.P. Vigier, Lett. Nuovo Cimento 26 (1979) 149; Phys. Lett. 88A (1982) 272. 
[13] Kh. Namsrai, J. Phys. A14 (1981) 1307; Sov. J. Part. Nucl. 12 (1981) 449.

[14] Ph. Droz-Vincent, Ann. Inst. Henri Poincaré 27 (1977) 407; Phys. Rev. D19 (1979) 702;

N. Cufaro Petroni, Ph. Droz-Vincent and J.P. Vigier, Lett. Nuovo Cimento 31 (1981) 415.

[15] N. Cufaro Petroni and J.P. Vigier, Causal action-at-a-distance and a new possible deduction of quantum mechanics from general relativity: the many body problem, Nuovo Cimento B, to be published.

[16] A. Garuccio, V.A. Rapisarda and J.P. Vigier, Lett. Nuovo Cimento 32 (1981) 451.

[17] S.J. Freedman and F. Clauser, Phys. Rev. Lett. 28 (1972) 938;

R.A. Holt and F.M. Pipkin, Harvard preprint (1974), unpublished;

G. Faraci, S. Gutkowsky, S. Notarrigo and A.R. Pennisi, Lett. Nuovo Cimento 9 (1974) 667;

L. Kasday, J. Ullman and C.S. Wu, Nuovo Cimento B25 (1975) 663;

J.F. Clauser, Phys. Rev. Lett. 36 (1976) 1223;

E.S. Fry and R.C. Thompson, Phys. Rev. Lett. 37 (1976) 465;

A.R. Wilson, J. Lowe and D.K. Butt, J. Phys. G2 (1976) 613;

M. Bruno, M. D'Agostino and C. Maroni, Nuovo Cimento B40 (1977) 142 .
[18] J.S. Bell, Physics 1 (1964) 195;

J.F. Clauser, M.A. Horne, A. Shimony and R.A. Holt, Phys. Rev. Lett. 23 (1969) 880.

[19] A. Aspect, Phys. Lett. 54A (1975) 117; Progr. Sci. Cult. 1 (1976) 439; Phys. Rev. D14 (1978) 1944.

[20] O. Costa de Beauregard, Physis (Florence) 22 (1981) 317.

[21] R.P. Feynman, Int. J. Theor. Phys. 21 (1982) 467.

[22] L. de Broglie, Une interprétation causale et non linéaire de la méchanique quantique (Paris, 1972).

[23] F. Halbwachs, Théorie relativiste des fluides à spin (Paris, 1960).

[24] J.P. Vigier, Lett. Nuovo Cimento 29 (1980) 467; Astron. Nachr. 303 (1982) 55.

[25] Y. Aharonov and D. Bohm, Phys. Rev. 115 (1959) 485; 123 (1961) $1511 ; 125$ (1962) 2192; 130 (1963) 1625; C. Philippidis and D. Bohm, The Aharonov-Bohm effect and the quantum potential, Nuovo Cimento $B$, to be published.

[26] E.P. Wigner, Symmetries and reflections (MIT Press, Cambridge, MA, 1971).

[27] H.P. Stapp, Nuovo Cimento B40 (1977) 151.

[28] D. Bohm and B. Hiley, Found. Phys. 5 (1975) 93.

[29] A. Garuccio, K.R. Popper and J.P. Vigier, Phys. Lett. 86A (1981) 397;

A. Garuccio, V.A. Rapisarda and J.P. Vigier, Phys. Lett. 90A (1982) 17.

[30] A. Gozzini, Comm. at the Symp, on Wave-particle dualism (Perugia, 1982). 